\title{
ON THE SYMMETRIC ENVELOPING ALGEBRA OF PLANAR ALGEBRA SUBFACTORS
}

\author{
STEPHEN CURRAN, VAUGHAN F. R. JONES, AND DIMITRI SHLYAKHTENKO
}

\begin{abstract}
We give a diagrammatic description of Popa's symmetric enveloping algebras associated to planar algebra subfactors. As an application we construct a natural family of derivations on these factors and compute a certain free entropy dimension type quantity.
\end{abstract}

\section{INTRODUCTION}

Inspired by Voiculescu's [19] description of the large $N$ limit of the distribution of families of independent random matrices, Guionnet, Jones and Shlyakhtenko [7] introduced diagrammatic traces on a tower of graded algebras $G r_{0}(\mathcal{P}) \subset G r_{1}(\mathcal{P}) \subset$ ... which is naturally associated to a subfactor planar algebra $\mathcal{P}$. The main result of [7] is that the completions form a tower of $\mathrm{II}_{1}$ factors $M_{0} \subset M_{1} \subset \cdots$ whose standard invariant is $\mathcal{P}$, thus giving a diagrammatic proof of a fundamental result of Popa [15]. In subsequent work [8] they were able to identify the isomorphism class of these factors in the case that $\mathcal{P}$ is finite-depth. Explicitly, $M_{k}$ is isomorphic to an interpolated free group factor $L\left(\mathbb{F}_{r_{k}}\right)$, where

$$
r_{k}=1+2 \delta^{-2 k} I(\delta-1) \text {. }
$$

Here $\delta^{2}$ is the index $\left[M_{1}: M_{0}\right]$; see Section 3 for the definition of the global in$\operatorname{dex} I$. The fact that $M_{k}$ is an interpolated free group factor was also obtained independently by Kodiyalam and Sunder [12. See also [9], [10, [11.

Given an inclusion of $\mathrm{II}_{1}$ factors $N \subset M$, Popa 14 has shown that there is a unique $\mathrm{II}_{1}$ factor $M \otimes_{e_{N}} M^{o p}$, called the symmetric enveloping algebra of the inclusion, which is generated by a copy of $M \otimes M^{o p}$ and a projection $e_{N}$ which is simultaneously the Jones projection for the inclusions $N \subset M$ and $N^{o p} \subset M^{o p}$. This algebra, together with the inclusion $M \otimes M^{o p} \subset M \otimes_{e_{N}} M^{o p}$, encodes a number of important analytic properties of the subfactor $N \subset M$, such as amenability and property $\mathrm{T}$ [16]. When the inclusion $N \subset M$ is strongly amenable, for example if $M$ is hyperfinite and $N \subset M$ is finite-depth, then $M \otimes M^{o p} \subset M \otimes_{e_{N}} M^{o p}$ is isomorphic to Ocneanu's asymptotic inclusion [13].

In this paper we give a diagrammatic construction of the symmetric enveloping algebras of the inclusions $M_{k} \subset M_{k+1}$. (In the case that $\mathcal{P}$ is the Temperley-Lieb planar algebra; this was announced in [17.) Using this description, we give a simple

Received by the editors October 3, 2011.

2010 Mathematics Subject Classification. Primary 46L37, 46 L54.

The first author's research was supported by an NSF postdoctoral fellowship and NSF grant DMS-0900776.

The second author's research was supported by NSF grant DMS-0856316.

The third author's research was supported by NSF grant DMS-0900776.

All the authors were also supported by DARPA Award 0011-11-0001. 
diagrammatic proof that the index $\left[M_{k} \otimes_{e_{k-1}} M_{k}^{o p}: M_{k} \otimes M_{k}^{o p}\right]$ is the global index $I$, first computed by Ocneanu [13. As an application we construct a module of closable derivations $\delta: G r_{0} \rightarrow G r_{0} \otimes G r_{0}^{o p}$, related to the Schwinger-Dyson equation from [9], whose Murray-von Neumann dimension (after completion) is precisely the parameter $r_{0}$ from $(*)$. Since the dimensions of such spaces of derivations are wellknown to be related to Voiculescu's free entropy dimension (see e.g. [18]), this provides a more conceptual understanding of the rather mysterious parameters $r_{k}$ computed in 8 .

Our paper is organized as follows. We begin by briefly recalling the construction from [7] in Section 1, In Section 2 we construct an auxiliary semi-finite algebra $\mathfrak{M}$, starting from a subfactor planar algebra $\mathcal{P}$. Section 3 contains our main result: the symmetric enveloping algebras of the inclusions $M_{k} \subset M_{k+1}$ are naturally isomorphic to certain compressions of $\mathfrak{M}$. We then compute the index of $M_{k} \otimes$ $M_{k}^{o p} \subset M_{k} \otimes_{e_{k-1}} M_{k}^{o p}$. In Section 4 we construct derivations on $G r_{0}$ and compute a free entropy dimension type quantity for $M_{0}$.

\section{Planar algebra subfactors}

In this section we briefly recall the construction from 7 . The reader is referred to this paper and to [10, 8] for further details.

Let $\mathcal{P}$ be a subfactor planar algebra with graded components $P_{k}^{\epsilon}, k=0,1,2, \ldots$, $\epsilon= \pm$. For $n, k \geq 0$ and $\epsilon= \pm$ let $P_{n, k}^{\epsilon}$ be a copy of $P_{n+k}^{\epsilon}$. Elements of $P_{n, k}^{\epsilon}$ will be represented by diagrams

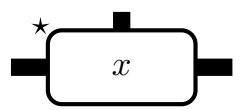

where the thick lines to the left and right represent $k$ strings, and the thick line at the top represents $2 n$ strings. We will typically suppress the marked point $\star$, and take the convention that it occurs at the top-left corner and is shaded according to $\epsilon$.

Define a product $\wedge_{k}: P_{n, k}^{\epsilon} \times P_{m, k}^{\epsilon} \rightarrow P_{n+m, k}^{\epsilon}$ by

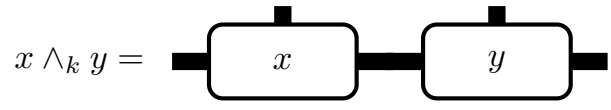

The involution $\dagger: P_{n, k}^{\epsilon} \rightarrow P_{n, k}^{\epsilon}$ is given by

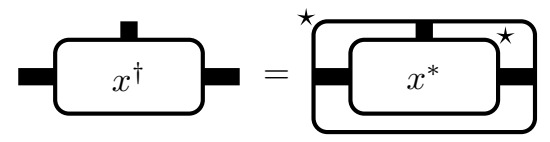

The Voiculescu trace $\tau_{k}: P_{n, k}^{\epsilon} \rightarrow \mathbb{C}$ is defined by

$$
\tau_{k}(x)=\delta^{-k} \cdot \underbrace{\sum T L}_{x}
$$

where $\sum T L$ denotes the sum over all loopless Temperley-Lieb diagrams with $2 n$ boundary points. Let $G r_{k}^{\epsilon}(\mathcal{P})=\bigoplus_{n \geq 0} P_{n, k}^{\epsilon}$, and observe that the formulas above 
give $G r_{k}^{\epsilon}(\mathcal{P})$ the structure of a graded $*$-algebra with trace $\tau_{k}$. The unit of $G r_{k}^{\epsilon}(\mathcal{P})$ is the element of $P_{0, k}^{\epsilon}$ consisting of $k$ parallel lines.

Let $\mathbf{e}_{k, \epsilon}$ denote the following element of $P_{0, k+2}^{\epsilon}, k \geq 0$ :

$$
\mathbf{e}_{k, \epsilon}=\longdiv { S }
$$

Note that there are natural inclusions of $G r_{k}^{\epsilon}(\mathcal{P})$ into $G r_{k+1}^{\epsilon}(\mathcal{P})$ defined by

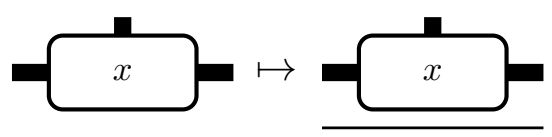

The main result of [7] is the following:

Theorem 1.1. For $k \geq 0$ and $\epsilon= \pm$, the Voiculescu trace $\tau_{k}$ is a faithful tracial state on $G r_{k}^{\epsilon}(\mathcal{P})$, and its $G N S$ completion is a $I I_{1}$ factor $M_{k}^{\epsilon}$ as long as $\delta>1$. The inclusions $G r_{k}^{\epsilon}(\mathcal{P}) \subset G r_{k+1}^{\epsilon}(\mathcal{P})$ extend to $M_{k}^{\epsilon} \subset M_{k+1}^{\epsilon}$, and $\left(M_{k+1}^{\epsilon}, \mathbf{e}_{k, \epsilon}\right)$ is the tower of the basic construction for the subfactor $M_{0}^{\epsilon} \subset M_{1}^{\epsilon}$. Moreover, the planar algebra of $M_{0} \subset M_{1}$ is isomorphic to $\mathcal{P}$.

\section{A Semi-Finite enveloping Algebra Associated to A Planar Algebra}

Let $\mathcal{P}$ be a subfactor planar algebra. For integers $k_{1}, k_{2}, s, t$ which add to $2 n$, and $\epsilon= \pm$, let $V_{k_{1}, k_{2}}^{\epsilon}(s, t)$ be a copy of $P_{n}^{\epsilon}$. Elements of $V_{k_{1}, k_{2}}^{\epsilon}(s, t)$ will be represented by diagrams of the form

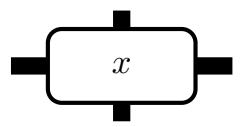

where the thick lines at the left, right, top and bottom represent $k_{1}, k_{2}, s$ and $t$ strings, respectively. We take the convention that the marked point occurs at the upper left corner, which is shaded $\epsilon$. If $\epsilon$ is not specified we assume that $\epsilon=+$.

Define a product $\wedge: V_{k_{1}, k_{2}}^{\epsilon}(s, t) \times V_{k_{1}^{\prime}, k_{2}^{\prime}}^{\epsilon^{\prime}}\left(s^{\prime}, t^{\prime}\right) \rightarrow V_{k_{1}, k_{2}^{\prime}}^{\epsilon}\left(s+s^{\prime}, t+t^{\prime}\right)$ to be zero unless $k_{2}=k_{1}^{\prime}$ and $\epsilon^{\prime}=(-1)^{s} \cdot \epsilon$, and otherwise by

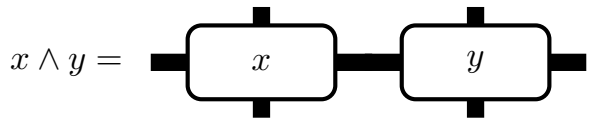

The adjoint $\dagger: V_{k_{1}, k_{2}}^{\epsilon}(s, t) \rightarrow V_{k_{2}, k_{1}}^{(-1)^{s} \epsilon}(s, t)$ is defined by

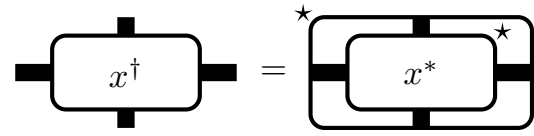

Define $\operatorname{Tr}: V_{k_{1}, k_{2}}^{\epsilon}(s, t) \rightarrow \mathbb{C}$ to be zero unless $k_{1}=k_{2}$ and both $s$ and $t$ are even, and otherwise by

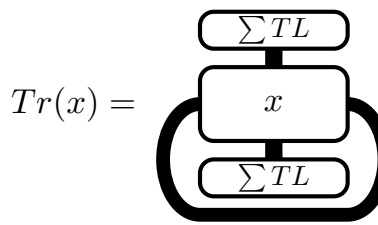


Let

$$
V=\bigoplus_{\epsilon= \pm} \bigoplus_{\begin{array}{c}
k_{1}, k_{2}, s, t \geq 0 \\
k_{1}+k_{2}+s+t \text { even }
\end{array}} V_{k_{1}, k_{2}}^{\epsilon}(s, t) .
$$

It is easy to see that the formulas above give $V$ the structure of a (non-unital) graded $*$-algebra with trace $\operatorname{Tr}$.

Theorem 2.1. $\mathrm{Tr}$ is a faithful, positive linear functional on $V$. Moreover, $V$ acts by bounded operators on the GNS Hilbert space for Tr.

The (semi-finite) von Neumann algebra obtained by completing $V$ under the GNS representation for $\operatorname{Tr}$ will be denoted $\mathfrak{M}$. To prove Theorem 2.1 we use the orthogonalization method of [10].

2.1. An orthogonal basis. We will now define a new multiplication and trace on $V$. To distinguish between these two structures, we define $W_{k_{1}, k_{2}}^{\epsilon}(s, t)$ to be a copy of $V_{k_{1}, k_{2}}^{\epsilon}(s, t)$, and let $W$ be the same direct sum defining $V$.

Define a product $\star$ on $W_{k_{1}, k_{2}}^{\epsilon}(s, t) \times W_{k_{1}^{\prime}, k_{2}^{\prime}}^{\epsilon^{\prime}}\left(s^{\prime}, t^{\prime}\right)$ to be zero unless $k_{2}=k_{1}^{\prime}$ and $\epsilon^{\prime}=(-1)^{s} \cdot \epsilon$, and otherwise by

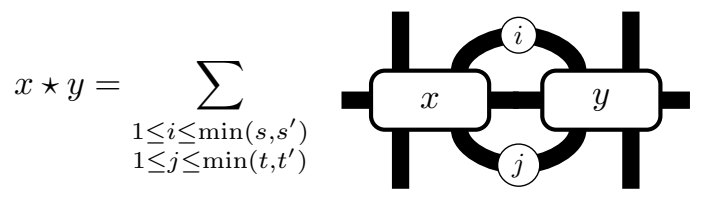

where $i$ and $j$ mean that there are $i$ parallel strings at the top and $j$ parallel strings at the bottom. The numbers of the other parallel strings are then determined by our conventions.

Define a trace $\operatorname{Tr}^{\prime}: W_{k_{1}, k_{2}}^{\epsilon}(s, t)$ to be zero unless $k_{1}=k_{2}$ and $s=t=0$, and otherwise by

$$
\operatorname{Tr}^{\prime}(x)=x
$$

Observe that there is a natural action of $T L \otimes T L^{o p}$ on $V$ as follows: if $a, b$ are TL diagrams with $s^{\prime}$ (resp. $t$ ) points on the top and $s$ (resp. $t^{\prime}$ ) points on the bottom, then $a \otimes b$ acts as the sum over $k_{1}, k_{2}$ and $\epsilon$ of the linear maps $V_{k_{1}, k_{2}}^{\epsilon}(s, t) \rightarrow$ $V_{k_{1}, k_{2}}^{\epsilon}\left(s^{\prime}, t^{\prime}\right)$ defined by

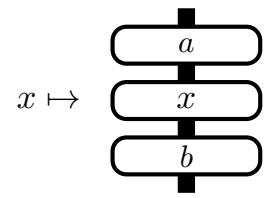

Recall that a TL diagram is called epi if each point on the top is connected to a point on the bottom, and monic if each point on the bottom is connected to a point on the top. Furthermore, an epi (resp. monic) TL diagram is said to be non-nested if each "turn-back" on the bottom (resp. top) of the diagram encloses no other turn-backs.

Define $X: V \rightarrow W$ to be the sum of all $a \otimes b$ where $a$ (resp. $b$ ) are epi (resp. monic) TL diagrams. Likewise, define $Y: W \rightarrow V$ to be the sum of all $a \otimes b$ where 
$a$ (resp. b) are non-nested epi (resp. monic) TL diagrams, and the coefficient on $a \otimes b$ is $(-1)^{s+t-s^{\prime}-t^{\prime}}$ when $a, b$ are as above.

\section{Proposition 2.2.}

(1) $X Y=1=Y X$.

(2) $X(a \wedge b)=X(a) \star X(b)$.

(3) $X\left(a^{*}\right)=X(a)^{*}$.

(4) $\operatorname{Tr}^{\prime}(X(a))=\operatorname{Tr}(a)$.

Proof. The proof is identical to the argument in [10, Section 5], and will therefore be omitted.

Corollary 2.3. $(W, \star, *)$ is an associative $*$-algebra.

Proof. Since $(V, \wedge, *)$ is clearly associative, this follows from the isomorphism in Proposition 2.2

We are now prepared to prove Theorem 2.1 .

Proof of Theorem 2.1. By Proposition 2.2, to prove the theorem it suffices to show that $T r^{\prime}$ is a faithful, positive state on $W$ and that $W$ acts by bounded operators on the GNS Hilbert space for $T r^{\prime}$. That $T r^{\prime}$ is positive and faithful is one of the defining axioms of the subfactor planar algebra $\mathcal{P}$. The fact that $W$ acts by bounded operators on the associated GNS Hilbert space may be proved along the same lines as [10, Theorem 3.3].

\section{The SYMmetric ENVELOPING ALGEBRA}

For $k \geq 0$ and $\epsilon= \pm$, let $p_{k, \epsilon} \in V_{2 k, 2 k}^{\epsilon}(0,0)$ be the diagram consisting of $2 k$ parallel strings, which is a projection in $V$. Define $G r_{k}^{\epsilon}(\mathcal{P}) \otimes G r_{k}^{\epsilon}(\mathcal{P})$ to be the compression $p_{k, \epsilon} V p_{k, \epsilon}$, i.e.

$$
G r_{k}^{\epsilon}(\mathcal{P}) \otimes G r_{k}^{\epsilon}(\mathcal{P})=\bigoplus_{k, s, t \geq 0} V_{2 k, 2 k}^{\epsilon}(2 s, 2 t)
$$

Define $\tau_{k} \otimes \tau_{k}$ on $G r_{k}^{\epsilon}(\mathcal{P}) \otimes G r_{k}^{\epsilon}(\mathcal{P})$ to be $\delta^{-2 k}$ times the restriction of $\operatorname{Tr}$.

It follows from Theorem 2.1 that $\tau_{k} \otimes \tau_{k}$ is a faithful, tracial state on $G r_{k}^{\epsilon}(\mathcal{P}) \otimes$ $G r_{k}^{\epsilon}(\mathcal{P})$. Let $M_{k}^{\epsilon} \otimes M_{k}^{\epsilon}$ denote its GNS completion, which is naturally identified with the compression $p_{k, \epsilon} \mathfrak{M} p_{k, \epsilon}$.

As an example, consider the element ||$\in V_{0,0}^{+}(2,2) \subset M_{0} \otimes M_{0}$ which consists of 2 vertical lines. We will show that the moments of || are related to the famous meander problem (see e.g. 3]). Recall that a (generalized) meander of order $n$ is a planar configuration of closed, non-intersecting loops crossing an infinite oriented line through $2 n$ points. Let $M_{n}^{(k)}$ denote the number of meanders of order $n$ with $k$ connected components, and define the meander polynomials $m_{n}(q)$ by

$$
m_{n}(q)=\sum_{k=0}^{n} M_{n}^{(k)} q^{k} .
$$

Proposition 3.1. If $\mathcal{P}$ is a subfactor planar algebra of modulus $\delta$, then

$$
\tau_{0} \otimes \tau_{0}\left((||)^{n}\right)=m_{n}(\delta) .
$$


Proof. We have

$$
\tau_{0} \otimes \tau_{0}\left((||)^{n}\right)=\frac{\sum T L}{2(2 n}
$$

As explained in [3, Section 3.3], this is precisely $m_{n}(\delta)$.

We will now show that $M_{k} \otimes M_{k}$ is naturally identified with Popa's symmetric enveloping algebra $M_{k} \bigotimes_{e_{k-1}} M_{k}^{o p}$. First note that there is a natural antiautomorphism $y \mapsto y^{o p}$ of $G r_{k}^{\epsilon}(\mathcal{P}) \otimes G r_{k}^{\epsilon}(\mathcal{P})$, determined by

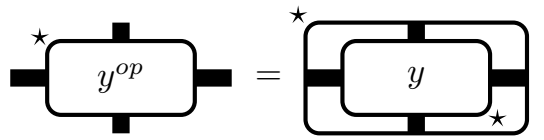

Since $y \mapsto y^{o p}$ preserves the trace $\tau_{k} \otimes \tau_{k}$, this extends to an anti-automorphism of $M_{k}^{\epsilon} \otimes M_{k}^{\epsilon}$.

Observe also that $G r_{k}^{\epsilon}(\mathcal{P}) \otimes G r_{k}^{\epsilon}(\mathcal{P})$ contains a copy of $G r_{k}^{\epsilon}(\mathcal{P}) \otimes G r_{k}^{\epsilon}(\mathcal{P})^{o p}$ as follows:

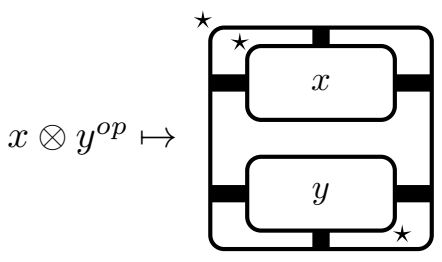

Since $\tau_{k} \otimes \tau_{k}$ restricts to the Voiculescu trace on $G r_{k}^{\epsilon}(\mathcal{P}) \otimes G r_{k}^{\epsilon}(\mathcal{P})^{o p}$, this inclusion extends to $M_{k}^{\epsilon} \otimes\left(M_{k}^{\epsilon}\right)^{o p} \subset M_{k}^{\epsilon} \otimes M_{k}^{\epsilon}$. Finally, if $k \geq 1$ and $\mathbf{f}_{k, \epsilon} \in M_{k}^{\epsilon} \otimes M_{k}^{\epsilon}$ is the projection

$$
\mathbf{f}_{k, \epsilon}=\delta^{-1} \cdot \underbrace{(k-1)}
$$

then $\mathbf{f}_{k,+}$ implements the Jones projections for both $M_{k-1} \subset M_{k}$ and $M_{k-1}^{o p} \subset M_{k}^{o p}$. It now follows from [14, Theorem 1.2] and the discussion above that $W^{*}\left(M_{k} \otimes\right.$ $\left.M_{k}^{o p}, \mathbf{f}_{k}\right)$ is isomorphic to $M_{k} \bigotimes_{e_{k-1}} M_{k}^{o p}$; in particular, it is a factor. We will now show that $M_{k} \otimes M_{k}=W^{*}\left(M_{k} \otimes M_{k}^{o p}, \mathbf{f}_{k}\right)$. First we need a lemma.

Let $k \geq 0$ and $\epsilon= \pm$, and define $c_{k, \epsilon} \in V_{2 k, 2 k+2}^{\epsilon}(1,1)$ by

$$
c_{k, \epsilon}=2
$$

It follows from [7, Lemma 2] that $c_{k, \epsilon} c_{k, \epsilon}^{*}$ is invertible in $M_{k}^{\epsilon} \otimes M_{k}^{\epsilon}$. Let $q_{k, \epsilon}$ be the initial projection of $c_{k, \epsilon}^{*} c_{k, \epsilon}$,

$$
q_{k, \epsilon}=c_{k, \epsilon}^{*}\left(c_{k, \epsilon} c_{k, \epsilon}^{*}\right)^{-1} c_{k, \epsilon} .
$$

Lemma 3.2. Fix $k \geq 0$ and $\epsilon= \pm$.

(1) The map

$$
\theta_{k, \epsilon}(x)=c_{k, \epsilon}^{*}\left(c_{k, \epsilon} c_{k, \epsilon}^{*}\right)^{-1 / 2} x\left(c_{k, \epsilon} c_{k, \epsilon}^{*}\right)^{-1 / 2} c_{k, \epsilon}
$$

is an isomorphism of $M_{k}^{\epsilon} \otimes M_{k}^{\epsilon}$ onto $q_{k, \epsilon}\left(M_{k+1}^{-\epsilon} \otimes M_{k+1}^{-\epsilon}\right) q_{k, \epsilon}$. 
(2) If $k \geq 1$, then $\theta_{k, \epsilon}$ restricts to an isomorphism

$$
W^{*}\left(M_{k}^{\epsilon} \otimes\left(M_{k}^{\epsilon}\right)^{o p}, \mathbf{f}_{k, \epsilon}\right) \simeq q_{k, \epsilon}\left(W^{*}\left(M_{k+1}^{-\epsilon} \otimes\left(M_{k+1}^{-\epsilon}\right)^{o p}, \mathbf{f}_{k+1,-\epsilon}\right)\right) q_{k, \epsilon} .
$$

Proof. (1) is straightforward; the inverse of $\theta_{k, \epsilon}$ is given by

$$
y \mapsto\left(c_{k, \epsilon} c_{k, \epsilon}^{*}\right)^{-1 / 2} c_{k, \epsilon} y c_{k, \epsilon}^{*}\left(c_{k, \epsilon} c_{k, \epsilon}^{*}\right)^{-1 / 2} .
$$

For (2), since it is clear that $\theta_{k, \epsilon}$ takes $M_{k}^{\epsilon} \otimes\left(M_{k}^{\epsilon}\right)^{o p}$ onto $q_{k, \epsilon}\left(M_{k+1}^{-\epsilon} \otimes\left(M_{k+1}^{-\epsilon}\right)^{o p}\right) q_{k, \epsilon}$ and $\mathbf{f}_{k, \epsilon}$ to $q_{k, \epsilon} \mathbf{f}_{k+1,-\epsilon} q_{k, \epsilon}$, it suffices to show that $q_{k, \epsilon} W^{*}\left(M_{k+1}^{-\epsilon} \otimes\left(M_{k+1}^{-\epsilon}\right)^{o p}\right.$, $\left.\mathbf{f}_{k+1,-\epsilon}\right) q_{k, \epsilon}$ is generated by $q_{k}\left(M_{k+1}^{-\epsilon} \otimes\left(M_{k+1}^{-\epsilon}\right)^{o p}\right) q_{k, \epsilon}$ and $q_{k, \epsilon} \mathbf{f}_{k+1,-\epsilon} q_{k, \epsilon}$. Note that $q_{k, \epsilon}$ is contained in the $I I_{1}$ factor $M_{1}^{\epsilon} \otimes\left(M_{1}^{\epsilon}\right)^{o p}$, so there are partial isometries $v_{1}, \ldots, v_{n} \in M_{1}^{\epsilon} \otimes\left(M_{1}^{\epsilon}\right)^{o p}$ such that $v_{i}^{*} v_{i} \leq q_{k, \epsilon}$ and $\sum_{i} v_{i}^{*} q_{k, \epsilon} v_{i}=1$. Let $m_{1}, \ldots, m_{l} \in M_{k+1}^{\epsilon} \otimes\left(M_{k+1}^{-\epsilon}\right)^{o p}$. Then

$$
\begin{aligned}
q_{k, \epsilon}( & \left.m_{1} \mathbf{f}_{k+1,-\epsilon} m_{2} \cdots \mathbf{f}_{k+1,-\epsilon} m_{l}\right) q_{k, \epsilon} \\
= & q_{k, \epsilon} m_{1}\left(\sum_{i_{1}=1}^{n} v_{i_{1}}^{*} q_{k, \epsilon} v_{i_{1}}\right) \mathbf{f}_{k+1,-\epsilon} m_{2}\left(\sum_{i_{2}=1}^{n} v_{i_{2}}^{*} q_{k, \epsilon} v_{i_{2}}\right) \\
& \cdots m_{l-1}\left(\sum_{i_{l-1}=1}^{n} v_{i_{l-1}}^{*} q_{k, \epsilon} v_{i_{l-1}}\right) \mathbf{f}_{k+1,-\epsilon} m_{l} q_{k, \epsilon} \\
= & \sum_{1 \leq i_{1}, \ldots, i_{l-1} \leq n} q_{k, \epsilon}\left(m_{1} v_{i_{1}}^{*}\right) q_{k, \epsilon} \mathbf{f}_{k+1,-\epsilon} q_{k, \epsilon}\left(v_{i_{1}} m_{2} v_{i_{2}}^{*}\right) q_{k, \epsilon} \mathbf{f}_{k+1,-\epsilon} \\
& \cdots \mathbf{f}_{k+1,-\epsilon} q_{k, \epsilon}\left(v_{i_{l-1}} m_{l}\right) q_{k, \epsilon},
\end{aligned}
$$

where we have used the fact that $\mathbf{f}_{k+1,-\epsilon}$ commutes with $M_{1}^{-\epsilon} \otimes\left(M_{1}^{-\epsilon}\right)^{o p}$. The result follows.

Theorem 3.3. For any $k \geq 1$ we have

$$
M_{k} \otimes M_{k} \simeq M_{k} \otimes_{e_{k-1}} M_{k}^{o p} .
$$

Proof. As discussed above, we just need to show that $M_{k} \otimes M_{k}=W^{*}\left(M_{k} \otimes M_{k}^{o p}, \mathbf{f}_{k}\right)$. If $x \in G r_{k}(\mathcal{P}) \otimes G r_{k}(\mathcal{P})$, then by Lemma 3.2 we have $x \in W^{*}\left(M_{k} \otimes M_{k}^{o p}, \mathbf{f}_{k}\right)$ if $c_{k,+}^{*} x c_{k,+} \in W^{*}\left(M_{k+1}^{-} \otimes\left(M_{k+1}^{-}\right)^{o p}, \mathbf{f}_{k+1,-}\right)$. Iterating, it is enough to show that for $l$ sufficiently large we have

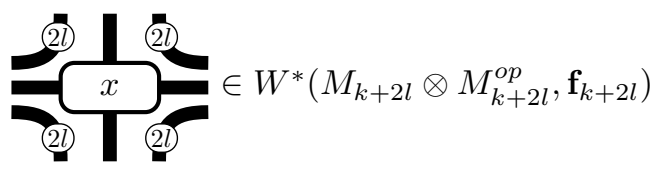

Let $x \in V_{2 k, 2 k}(2 s, 2 t)$. Then for $l=t+k$ we have that
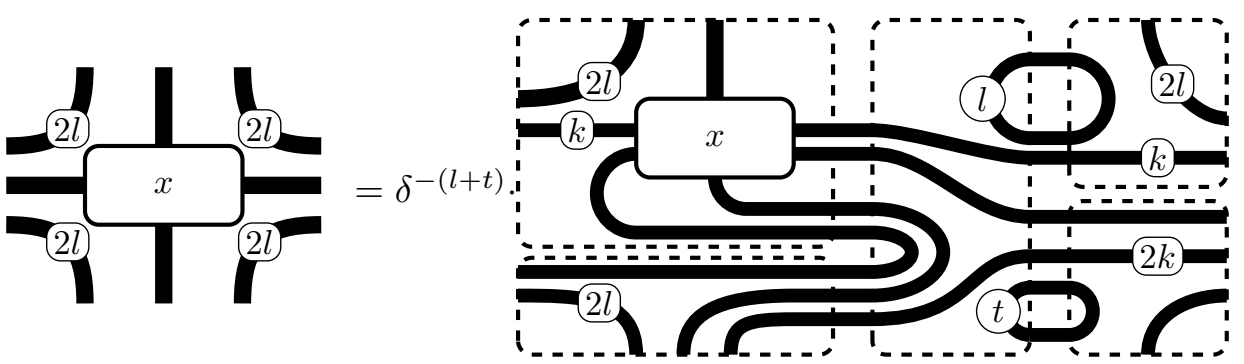
is the product of three elements in $M_{k+2 l} \otimes M_{k+2 l}$. The first and third terms are (manifestly) elements of $M_{k+2 l} \otimes M_{k+2 l}^{o p}$. The second term is a TemperleyLieb element, but since $W^{*}\left(M_{k+2 l} \otimes M_{k+2 l}^{o p}, \mathbf{f}_{k+2 l}\right)$ contains all of the standard Temperley-Lieb generators

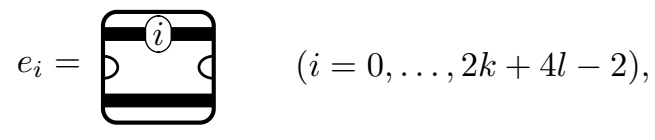

it contains any such Temperley-Lieb element. This completes the proof.

We will now give a diagrammatic computation of the index $\left[M_{0} \otimes M_{0}: M_{0} \otimes M_{0}^{o p}\right]$. Let $\Gamma$ be the principal graph of $\mathcal{P}$ (see e.g. [6]), and let $\Gamma_{+}$denote the collection of even vertices. Associated to each $v \in \Gamma_{+}$is an irreducible $M_{0}-M_{0}$ bimodule $X_{v}$. Let $*$ denote the distinguished vertex, corresponding to $X_{*}=L^{2}\left(M_{0}\right)$, and for $k \geq 0$ let $\Gamma_{+, k}$ denote the set of vertices in $\Gamma$ whose distance from $*$ is less than or equal to $2 k$. Then we have

$$
M_{0} L^{2}\left(M_{k}\right)_{M_{0}} \simeq \bigoplus_{v \in \Gamma_{+, k}} X_{v} \otimes \mathcal{H}_{v}(k)
$$

where $\mathcal{H}_{v}(k)$ are auxiliary finite-dimensional Hilbert spaces, whose dimensions we denote by $n_{k}(v)$.

It follows that

$$
M_{0} \otimes M_{0}^{o p} L^{2}\left(M_{k} \otimes M_{k}^{o p}\right)_{M_{0} \otimes M_{0}^{o p}} \simeq \bigoplus_{v, w \in \Gamma_{+, k}} X_{v} \otimes \overline{X_{w}} \otimes\left(\mathcal{H}_{v}(k) \otimes \overline{\mathcal{H}_{w}(k)}\right),
$$

where $\overline{X_{w}}$ is the contragredient bimodule and $\overline{\mathcal{H}_{w}(k)}$ is the conjugate Hilbert space. Let $1_{v}(k) \in \mathcal{H}_{v}(k) \otimes \overline{\mathcal{H}_{v}(k)}$ be the unit under the natural identification with $\operatorname{Hom}_{\mathbb{C}}\left(\mathcal{H}_{k}(v)\right)$, and define $p_{k}(v)$ to be the projection from $L^{2}\left(M_{k} \otimes M_{k}^{o p}\right)$ onto $X_{v} \otimes \overline{X_{v}} \otimes 1_{v}(k)$. Then define

$$
p_{k}=\sum_{v \in \Gamma_{+, k}} p_{k}(v)
$$

Recall that $P_{2 k}$ can be identified with $\operatorname{Hom}_{M_{0}, M_{0}}\left(L^{2}\left(M_{k}\right)\right)$; in particular, the central projections $q_{k}(v)$ of $P_{2 k}$ are indexed by $v \in \Gamma_{+, k}$. Let $t_{k}(v)$ denote the trace of a minimal projection in $P_{2 k}(v):=P_{2 k} \cdot q_{k}(v)$, the central component of $P_{2 k}$ corresponding to $v \in \Gamma_{+, k}$. If $\left\{e_{i j}(v): 1 \leq i, j \leq n_{k}(v)\right\}$ is a choice of matrix units for $P_{2 k}(v)$, then we have

$$
p_{k}(v)=\frac{1}{n_{k}(v)} \sum_{1 \leq i, j \leq n_{k}(v)} e_{i j}(v) \otimes e_{j i}(v)^{o p} .
$$

Graphically, we will represent $p_{k}(v)$ using a Sweedler type convention as follows:

$$
p_{k}(v)=\begin{aligned}
& p_{k}^{(1)}(v) \\
& p_{k}^{(2)}(v)
\end{aligned}
$$


Lemma 3.4. Let $x, y \in P_{2 k}$ and $v \in \Gamma_{+, k}$; then we have

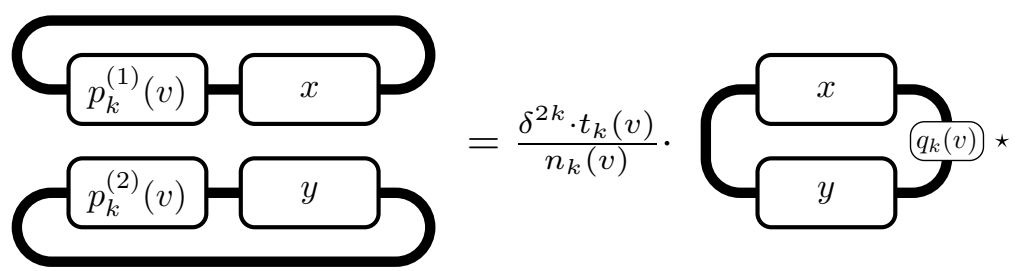

Proof. Let $\operatorname{Tr}_{v}$ denote the unnormalized trace on $P_{2 k}(v) \simeq M_{n_{k}(v)}(\mathbb{C})$, so that $\left.\tau_{k}\right|_{P_{2 k}(v)}=t_{k}(v) \cdot T r_{v}$. Then the left hand side of the formula in the statement is equal to

$$
\begin{aligned}
\frac{1}{n_{k}(v)} \sum_{1 \leq i, j \leq n_{k}(v)} \delta^{4 k} \cdot t_{v}(k)^{2} \operatorname{Tr}_{v}\left(e_{i j}(v) x\right) \operatorname{Tr}_{v}\left(y e_{j i}(v)\right) \\
=\frac{\delta^{2 k} \cdot t_{k}(v)}{n_{k}(v)} \sum_{1 \leq i, j \leq n_{k}(v)} \delta^{2 k} \cdot t_{k}(v) \operatorname{Tr}_{v}\left(x y q_{k}(v)\right),
\end{aligned}
$$

which is equal to the right hand side.

Define $\Psi_{k}: G r_{k} \otimes G r_{k}^{o p} \rightarrow G r_{0} \otimes G r_{0}$ by linear extension of

$$
\Psi_{k}\left(x \otimes y^{o p}\right)=\sum_{v \in \Gamma_{+, k}} \sqrt{\frac{t_{k}(v)}{n_{k}(v)}}
$$

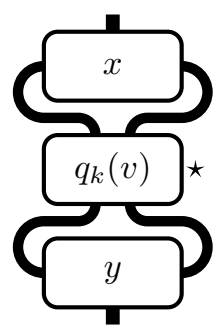

Theorem 3.5. $\Psi_{k}$ extends to an $M_{0} \otimes M_{0}^{o p}$-bilinear partial isometry from $L^{2}\left(M_{k} \otimes\right.$ $\left.M_{k}^{o p}\right)$ into $L^{2}\left(M_{0} \otimes M_{0}\right)$, whose initial projection is equal to $p_{k}$.

Proof. Let $x_{1}, x_{2}, y_{1}, y_{2} \in G r_{k}$; then $\left\langle\Psi_{k}\left(x_{1} \otimes y_{1}^{o p}\right), \Psi_{k}\left(x_{2} \otimes y_{2}^{o p}\right)\right\rangle$ is equal to

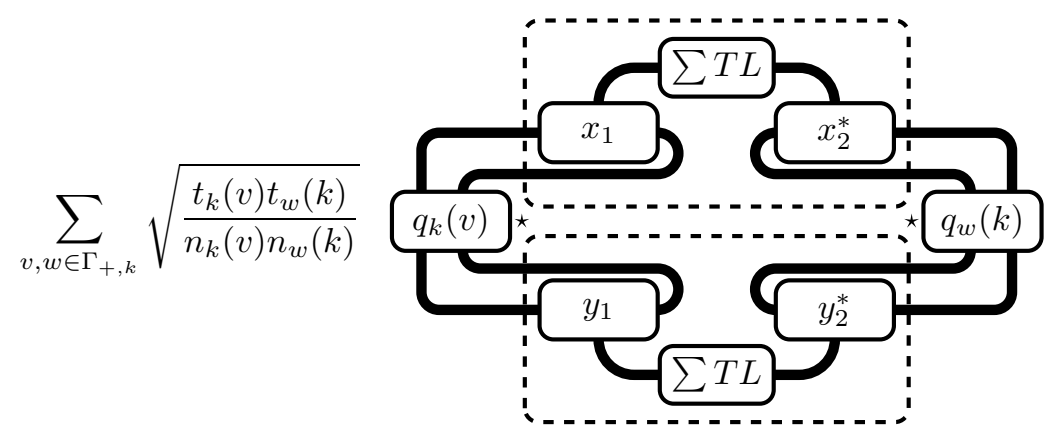

Let $x, y^{o p}$ denote the elements of $P_{2 k}, P_{2 k}^{o p}$ represented by the dashed boxes above. Since $q_{k}(v)$ and $q_{w}(k)$ are central, the terms in the sum above for which $v \neq w$ are equal to zero. But applying Lemma 3.4 to $x, y$, we see that the term corresponding 
to $v=w$ is equal to $\left\langle p_{k}(v) \cdot\left(x_{1} \otimes y_{1}^{o p}\right), x_{2} \otimes y_{2}^{o p}\right\rangle$. So we have proved that

$$
\left\langle\Psi_{k}\left(x_{1} \otimes y_{1}^{o p}\right), \Psi_{k}\left(x_{2} \otimes y_{2}^{o p}\right)\right\rangle=\left\langle p_{k} \cdot\left(x_{1} \otimes y_{1}^{o p}\right), x_{2} \otimes y_{2}^{o p}\right\rangle,
$$

from which the result follows.

From this we can deduce the following corollary, which is due to Ocneanu 13 . in the finite-depth case, and Popa [16] in the general case.

Corollary 3.6. We have

$$
M_{0} \otimes M_{0}^{o p} L^{2}\left(M_{0} \otimes M_{0}\right)_{M_{0} \otimes M_{0}^{o p}} \simeq \bigoplus_{v \in \Gamma_{+}} X_{v} \otimes \overline{X_{v}} .
$$

Proof. Let $\Xi_{k}$ denote the range of $\Psi_{k}$, which is a closed subspace of $L^{2}\left(M_{0} \otimes M_{0}\right)$ invariant under the left and right actions of $M_{0} \otimes M_{0}^{o p}$. By Theorem [3.5, $\Xi_{k}$ is isomorphic to

$$
\bigoplus_{v \in \Gamma_{+, k}} X_{v} \otimes \overline{X_{v}}
$$

as an $M_{0} \otimes M_{0}^{o p}$-bimodule. It follows that $\overline{\bigcup_{k \geq 0} \Xi_{k}}$ is isomorphic as an $M_{0} \otimes M_{0}^{o p}$ bimodule to

$$
\bigoplus_{v \in \Gamma_{+}} X_{v} \otimes \overline{X_{v}}
$$

Now if $x \in V_{0,0}(2 s, 2 t)$, then $x=\Psi_{t}\left(x_{t}\right)$, where $x_{t} \in M_{t} \otimes M_{t}^{o p}$ is defined by

$$
x_{t}=\sum_{v \in \Gamma_{+, t}} \sqrt{\frac{n_{k}(v)}{t_{k}(v)}} .
$$

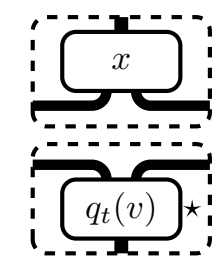

So $\bigcup_{k \geq 0} \Xi_{k}$ contains $G r_{0} \otimes G r_{0}$ and is therefore dense in $L^{2}\left(M_{0} \otimes M_{0}\right)$, which completes the proof.

Remark 3.7. Note that it follows from the proof of Corollary 3.6 that $\Psi_{k}$ is surjective if the depth of $\mathcal{P}$ is less than or equal to $2 k$, i.e. if $\Gamma_{+}=\Gamma_{+, k}$.

Corollary 3.8. Let $k \geq 0$. Then if $\Gamma_{+}$is finite the index $\left[M_{k} \otimes M_{k}: M_{k} \otimes M_{k}^{o p}\right]$ is equal to the global index

$$
I=\sum_{v \in \Gamma_{+}} \operatorname{dim}_{M_{0}}\left(X_{v}\right)^{2}
$$

Otherwise $\left[M_{k} \otimes M_{k}: M_{k} \otimes M_{k}^{o p}\right]=\infty$.

Proof. For $k=0$ this is clear from the previous corollary. The result then follows for $k \geq 1$ from Lemma 3.2 and the fact that the global index of $\mathcal{P}^{o p}$ (the planar algebra obtained from $\mathcal{P}$ by reversing the shading) is the same as that of $\mathcal{P}$. 


\section{Derivations on Planar Algebra FACTORS}

Throughout this section we will denote $A=G r_{0}(\mathcal{P})$ and $M=M_{0}(\mathcal{P})$, where $\mathcal{P}$ is a subfactor planar algebra. Define

$$
\Phi_{o}=\bigoplus_{s, t \geq 0} V_{1,0}^{-}(2 s+1,2 t), \quad \Phi_{e}=\bigoplus_{s, t \geq 0} V_{1,0}^{+}(2 s, 2 t+1), \quad \Phi=\Phi_{o} \oplus \Phi_{e} .
$$

Observe that $\Phi$ has a natural right $A \otimes A^{o p}$-module structure.

Given $Q \in \Phi_{o}$ and $x \in P_{n}$, define $\widetilde{\delta}_{Q}(x) \in A \otimes A$ by

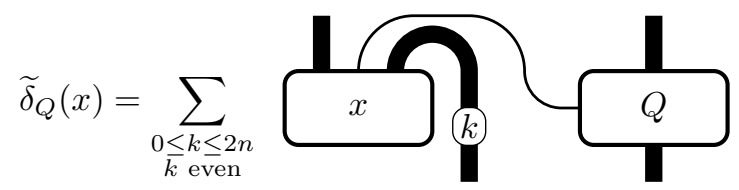

Extend $\widetilde{\delta}_{Q}$ linearly to obtain a map $\widetilde{\delta}_{Q}: A \rightarrow A \otimes A$. If $Q \in \Phi_{e}$ we define $\widetilde{\delta}_{Q}: A \rightarrow A \otimes A$ likewise, except that we sum over odd instead of even $k$ in the formula above. We then extend $Q \mapsto \widetilde{\delta}_{Q}$ linearly to $\Phi$.

Proposition 4.1. If $Q \in \Phi$, then $\widetilde{\delta}_{Q}$ is a derivation from $A$ into $A \otimes A$. The assignment $Q \mapsto \widetilde{\delta}_{Q}$ is an $A \otimes A^{o p}$-linear map from $\Phi$ into $\operatorname{Der}(A, A \otimes A)$.

Proof. For the first statement, it suffices to consider $Q \in \Phi_{o}$ or $Q \in \Phi_{e}$. In either case the result is clear from drawing the relevant diagram. The second statement is obvious.

Let $E_{M \otimes M^{o p}}$ denote the unique trace-preserving conditional expectation from $M \otimes M$ onto $M \otimes M^{o p}$, which is given by the orthogonal projection $L^{2}(M \otimes M) \rightarrow$ $L^{2}\left(M \otimes M^{o p}\right)$. We will now show that this projection may also be computed with respect to the orthogonal basis of Section 2.1] Let $\langle\langle\cdot, \cdot\rangle\rangle$ denote the inner product on $A \otimes A$ induced by $\delta^{-2 k}$ times the restriction of $\operatorname{Tr}^{\prime}$.

Lemma 4.2. Let $P$ denote the orthogonal projection of $A \otimes A$ onto $A \otimes A^{\text {op }}$ with respect to $\langle\langle\cdot, \cdot\rangle\rangle$. If $Q \in A \otimes A$, then

$$
E_{M \otimes M^{o p}}(Q)=P(Q) .
$$

Proof. SGM If $Q \in A \otimes A$ and $a \otimes b \in A \otimes A^{o p}$ we have

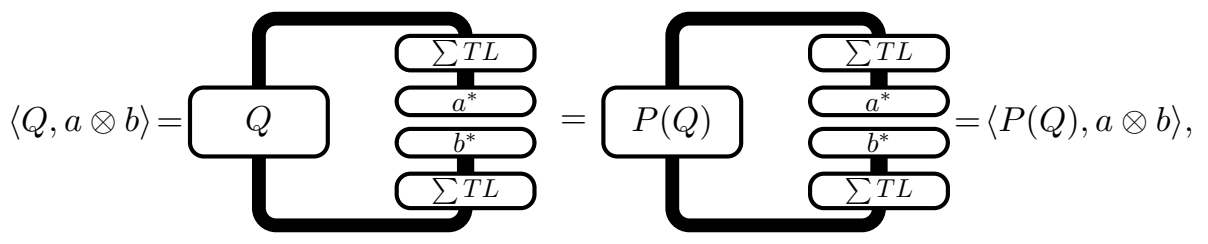

so that $P(Q)$ is equal to the orthogonal projection of $Q$ onto $M \otimes M^{o p}$ in $L^{2}\left(\tau_{k} \otimes \tau_{k}\right)$.

\section{Corollary 4.3.}

(1) $E_{M \otimes M^{o p}}\left(V_{0,0}^{+}(2 s, 2 t)\right) \subset P_{s} \otimes P_{t}$; in particular, $E_{M \otimes M^{o p}}(A \otimes A) \subset A \otimes A^{o p}$.

(2) Suppose that $Q \in A \otimes A \cap\left(A \otimes A^{o p}\right)^{\perp}$. Write

$$
Q=\sum_{s, t \geq 0} Q_{s, t}
$$


where $Q_{s, t} \in V_{0,0}^{+}(2 s, 2 t)$ are non-zero for finitely many $s, t \geq 0$. Then $Q_{s, t} \in\left(A \otimes A^{o p}\right)^{\perp}$ for all $s, t \geq 0$.

(3) If $Q \in V_{0,0}^{+}(2 s, 2 t) \cap\left(A \otimes A^{o p}\right)^{\perp}$, then

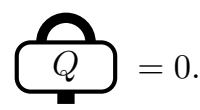

Proof. (1) and (2) are immediate from the previous lemma. Let $Q$ be as in (3). Then if $x \in P_{t}$ we have

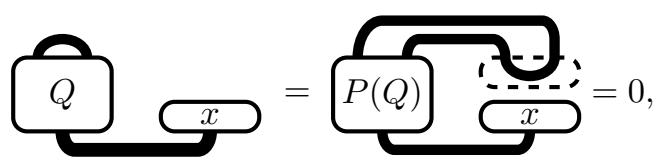

from which the result follows.

Given $Q \in \Phi$, define $\delta_{Q}=E_{M \otimes M o p} \circ \widetilde{\delta}_{Q}$. Note that the range of $\delta_{Q}$ is contained in $A \otimes A^{o p}$ by the previous corollary. Since $E_{M \otimes M^{o p}}$ commutes with the left and right actions of $A \otimes A^{o p}$, it is clear that $\delta_{Q} \in \operatorname{Der}\left(A, A \otimes A^{o p}\right)$ and that the assignment $Q \mapsto \delta_{Q}$ is $A \otimes A^{o p}$-linear.

We will now determine the kernel of the mapping $Q \mapsto \delta_{Q}$. Define

$$
\Omega_{o}=\bigoplus_{s, t \geq 0} V_{0,0}^{-}(2 s+1,2 t+1), \quad \Omega_{e}=\bigoplus_{s, t \geq 0} V_{0,0}^{+}(2 s, 2 t), \quad \Omega=\Omega_{o} \oplus \Omega_{e} .
$$

Observe that $\Omega_{e}=A \otimes A$; in particular, $A \otimes A^{o p} \subset \Omega$. The orthogonal complement of $A \otimes A^{o p}$ in $\Omega$ is $\Omega_{o} \oplus\left(A \otimes A^{o p}\right)^{\perp} \cap A \otimes A$.

Given $R \in \Omega_{o}$, define $\rho(R) \in \Phi$ by

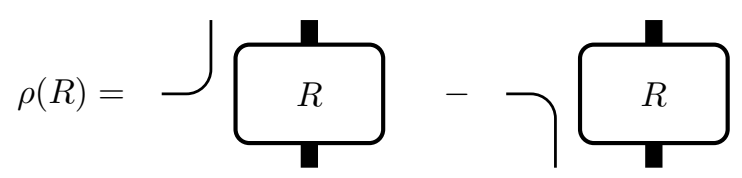

Likewise if $R \in \Omega_{e}$ we define $\rho(R) \in \Phi$ by the same formula, but note that the shading of the "corners" must be reversed. We then extend $\rho$ to $\Omega$ linearly.

Proposition 4.4. The restriction of $\rho$ to $\Omega \cap\left(A \otimes A^{o p}\right)^{\perp}$ is an $A \otimes A^{o p}$-linear isomorphism onto the kernel of the mapping $Q \mapsto \delta_{Q}$.

Proof. If $R \in \Omega_{o}$, then it is immediate from the defining formula that $\widetilde{\delta}_{\rho(R)}=0$. On the other hand, if $R \in \Omega_{e}$, then it is easy to see that $\widetilde{\delta}_{\rho(R)}(x)=[x, R]$, and therefore $\delta_{\rho(R)}(x)=\left[x, E_{M \otimes M^{o p}}(R)\right]$. So we have $\delta_{\rho(R)}=0$ if $R \perp A \otimes A^{o p}$. In fact since $M$ is diffuse, any Hilbert-Schmidt operator commuting with $A$ must be zero, and so we have $\delta_{\rho(R)}=0$ if and only if $R \perp A \otimes A^{o p}$.

It remains to show that if $Q \in \Phi$ is such that $\delta_{Q}=0$, then $Q=\rho(R)$ for a unique $R \in \Omega$. Write

$$
Q=\sum_{s, t \geq 0} Q_{s, t}
$$


where $Q_{s, t} \in V_{1,0}^{(-1)^{s}}(s, t)$ are non-zero for finitely many $s, t$. For $s, t \geq 1$ with $s+t$ even, define $R_{s, t} \in V_{0,0}^{(-1)^{s}}(s, t)$ by

$$
R_{s, t}=\sum_{k=1}^{\min \{s, t+1\}}
$$

Note that $R_{s, t}=0$ for all but finitely many $s, t$, and set $R=\sum_{s, t} R_{s, t} \in \Omega$. We claim that $Q=\rho(R)$; note that it will then follow from the discussion above that $R \in\left(A \otimes A^{o p}\right)^{\perp}$.

We need to show that for any $s, t \geq 0$ we have

$$
Q_{s, t}=-R_{R_{s-1, t}}^{\mathbf{R}}-R_{s, t-1}
$$

We will prove this by induction on $s$. First suppose that $s=0$; we claim that $Q_{s, t}=0$ for any $t \geq 0$. Indeed, this follows from $\delta_{Q}(\cup)=0$ and (2) of Corollary 4.3 .

So assume that $s \geq 1$. Let $x$ be the following Temperley-Lieb element with $2(s+1)$ boundary points:

$$
x=\varnothing
$$

Since $\delta_{Q}(x)=0$, applying (2) of Corollary 4.3 we find that

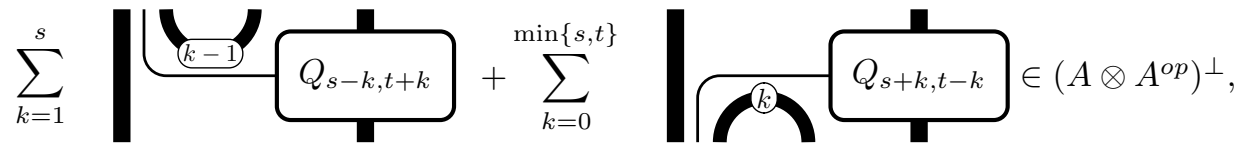

where the number of parallel lines appearing at left is determined by the condition that each term is in $V_{0,0}^{+}(2 s, t+s+1)$.

Applying induction to the first sum and simplifying, we are left with

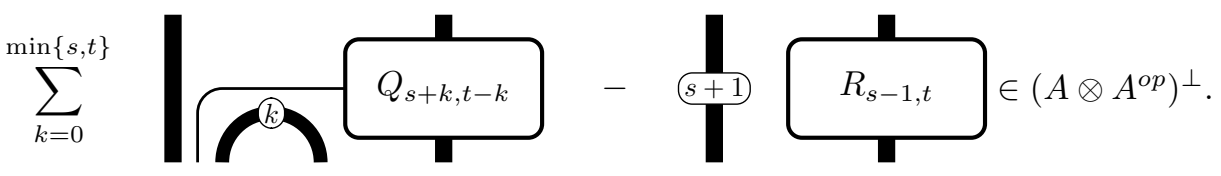

By (3) of Corollary 4.3, we have

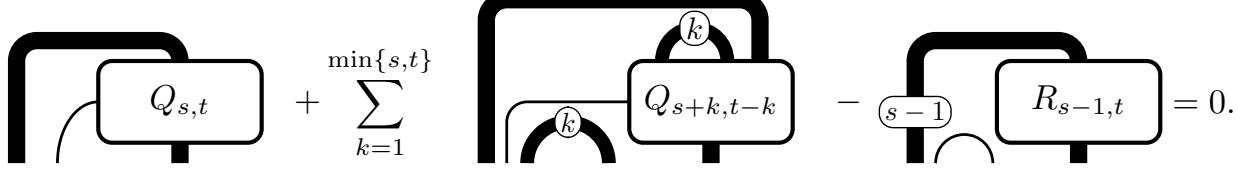

After rotating $s$ strings clockwise from bottom to top, and one more from the bottom to the left, we obtain the desired result.

Finally, if $Q=\rho\left(R^{\prime}\right)$ and $R$ is defined in terms of $Q$ as above, it is straightforward to check that $R=R^{\prime}$, which proves that $\rho$ is injective. Since $\rho$ is clearly linear over $A \otimes A^{o p}$, this completes the proof. 
We now compute the "conjugate variable" $\delta_{Q}^{*}(1 \otimes 1)$. This is closely related to the Schwinger-Dyson equation from [9, Lemma 12].

Proposition 4.5. Let $Q \in V_{1,0}^{(-1)^{s}}(s, t)$, and view $\delta_{Q}$ as a densely defined unbounded operator $\delta_{Q}: L^{2}(M) \rightarrow L^{2}\left(M \otimes M^{o p}\right)$. Then $1 \otimes 1 \in \mathfrak{D}\left(\delta_{Q}^{*}\right)$, and

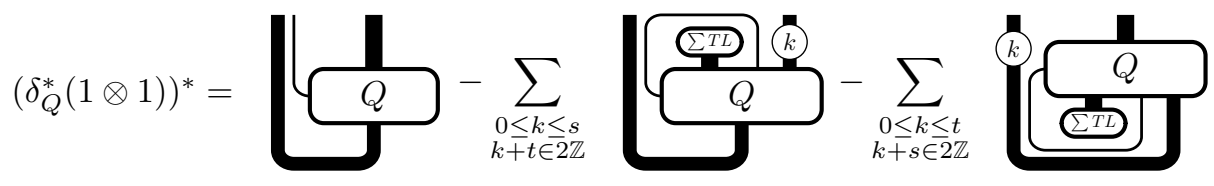

Proof. Fix $x \in A$ and look at

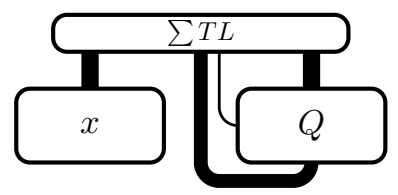

Consider the string on the left side of $Q$. For a fixed term in the sum over $T L$ diagrams there are three possibilities: this string is connected to the top of $Q$, to the bottom of $Q$, or to $x$. It follows that the inner product of $x$ with the element in the statement of the proposition is equal to the sum of the terms above in which the string on the left side of $Q$ is connected to $x$. But it is clear that this is equal to $\left(\tau_{k} \otimes \tau_{k}\right) \widetilde{\delta}_{Q}(x)=\left\langle\delta_{Q}(x), 1 \otimes 1\right\rangle$, which completes the proof.

Corollary 4.6. If $Q \in \Phi$, then $\delta_{Q}$ is closable as an unbounded operator $L^{2}(M) \rightarrow$ $L^{2}\left(M \otimes M^{o p}\right)$.

Proof. This follows from $1 \otimes 1 \in \mathfrak{D}\left(\delta_{Q}^{*}\right)$ by [20].

Note that $\Phi$ is identified with $\left(p_{1,+}+p_{1,-}\right) V p_{0,+}$, and so may be completed to obtain a Hilbert space $\bar{\Phi} \simeq\left(p_{1,+}+p_{1,-}\right) L^{2}(\mathfrak{M}) p_{0,+}$. Likewise $\Omega$ may completed to obtain a Hilbert space $\bar{\Omega} \simeq\left(p_{0,+}+p_{0,-}\right) L^{2}(\mathfrak{M}) p_{0,+}$.

Proposition 4.7. As right $M \otimes M^{o p}$ modules, we have

$$
\operatorname{dim}_{M \otimes M^{o p}}(\bar{\Phi})=2 \delta I
$$

and

$$
\operatorname{dim}_{M \otimes M^{o p}}(\bar{\Omega})=2 I .
$$

Proof. Let $c \in V_{0,1}^{-}(1,0)$ be the following element:

$$
c=\varnothing
$$

It follows from the same argument as in Lemma 3.2 that $L^{2}(M \otimes M) \simeq q \cdot \overline{\Phi_{o}}$ as right $M \otimes M^{o p}$-modules, where $q=c^{*}\left(c c^{*}\right)^{-1} c$ is the initial projection of $c^{*} c$. Note that there is an obvious inclusion of $M_{1}$ into the commutant of the right action of $M \otimes M^{o p}$ on $\bar{\Phi}_{o}$. It follows from the uniqueness of the trace on $M_{1}$ that the trace of $q$ in the commutant of $M \otimes M^{o p}$ is equal to its trace in $M_{1}$, which is $\delta^{-1}$. By Corollary 3.8 we then have

$$
\operatorname{dim}_{M \otimes M^{o p}} \overline{\Phi_{o}}=\delta \cdot I .
$$

Likewise we have $\operatorname{dim}_{M \otimes M^{o p}} \overline{\Phi_{e}}=\delta \cdot I$, so that $\operatorname{dim}_{M \otimes M^{o p}} \bar{\Phi}=2 \delta I$. 
Clearly we have $\overline{\Omega_{e}} \simeq L^{2}\left(M \otimes M^{o p}\right)$, and hence $\operatorname{dim}_{M \otimes M^{o p}} \overline{\Omega_{e}}=I$. On the other hand, let $c^{\prime} \in V_{0,1}^{+}(1,0)$ be as $c$ above but with the shading reversed, and set $q^{\prime}=c^{\prime *}\left(c c^{\prime *}\right)^{-1} c^{\prime}$. Then by the same argument as in Lemma 3.2, we have $\overline{\Omega_{o}} \simeq q^{\prime} \cdot \overline{\Phi_{e}}$, and hence $\operatorname{dim}_{M \otimes M^{o p}} \overline{\Omega_{o}}=I$.

Remark 4.8. For a family $X_{1}, \ldots, X_{n}$ of self-adjoint operators which generate a $\mathrm{II}_{1}$ factor $M$, Voiculescu has defined the free entropy dimension $\delta_{0}\left(X_{1}, \ldots, X_{n}\right)$. If $M$ is isomorphic to an interpolated free group factor $L \mathbb{F}_{t}$, it is generally expected (but not proven) that $\delta_{0}\left(X_{1}, \ldots, X_{n}\right)=t$.

Beginning with the work of Connes-Shlyakhtenko on $L^{2}$-Betti numbers [2], there have been a number of recent results relating Voiculescu's free entropy dimension to the Murray-von Neumann dimension of certain spaces of derivations. In particular, it it reasonable to expect that in "nice" situations, we should have the following equality:

$$
\operatorname{dim}_{M \otimes M^{o p}} \overline{\operatorname{Der}_{c}\left(A, A \otimes A^{o p}\right)}\left(L^{2}\left(M \otimes M^{o p}\right)\right)^{n}=\delta_{0}\left(X_{1}, \ldots, X_{n}\right),
$$

where $A=\mathbb{C}\left\langle X_{1}, \ldots, X_{n}\right\rangle$ and $\operatorname{Der}_{c}\left(A, A \otimes A^{o p}\right)$ denotes the module of closable derivations $\delta: A \rightarrow A \otimes A^{o p}$, which is embedded into $\left(L^{2}\left(M \otimes M^{o p}\right)\right)^{n}$ via $\delta \mapsto$ $\left(\delta\left(X_{1}\right), \ldots, \delta\left(X_{n}\right)\right)$. Note in particular that if $X_{1}, \ldots, X_{n}$ are free semi-circular random variables, then both sides of the equation are indeed equal to $n$.

Combining the results above, we have constructed an $A \otimes A^{o p}$-linear map from $D=\left\{\delta_{Q}: Q \in \Phi\right\}$ onto a dense subset of the orthogonal complement in $\bar{\Phi}$ of $\rho(\bar{\Omega})$. It follows that

$$
\operatorname{dim}_{M \otimes M^{o p}} \bar{D}^{\bar{\Phi}}=\operatorname{dim}_{M \otimes M^{o p}} \bar{\Phi}-\operatorname{dim}_{M \otimes M^{o p}} \rho\left(\bar{\Omega} \ominus L^{2}\left(M \otimes M^{o p}\right)\right) .
$$

One can show that the extension of $\rho$ to $\bar{\Omega}$ is still injective, but the proof is rather tedious and so we omit it. Assuming this, we are led to the equation

$$
\operatorname{dim}_{M \otimes M^{o p}} \bar{D}^{\bar{\Phi}}=2 \delta I-(2 I-1)=1+2 I(\delta-1)=r_{0} .
$$

Now if we have $D=\operatorname{Der}_{c}\left(A, A \otimes A^{o p}\right)$, then in light of the remark above this "explains" the formula for $r_{0}$ from [8]. Similar reasoning may of course be applied to the parameters $r_{k}, k \geq 1$.

So we are led to the question of whether every closable derivation $\delta: A \rightarrow A \otimes A^{\text {op }}$ is equal to $\delta_{Q}$ for some $Q \in \Phi$. First note that it is not the case that every (algebraic) derivation $A \rightarrow A \otimes A^{o p}$ is of this form. For example, when $\mathcal{P}$ is the tensor planar algebra on $\mathbb{C}^{n}$, then $A \simeq \mathbb{C}\left\langle t_{1}, \ldots, t_{n^{2}}\right\rangle$ is the free algebra generated by $P_{1}$. There are then the free difference quotient derivations $\partial_{i}: A \rightarrow A \otimes A^{o p}$ defined by

$$
\partial_{i}\left(t_{j}\right)=\delta_{i j} \cdot 1 \otimes 1 \text {. }
$$

Note that $\partial_{i}$ takes the $n$ graded component of $A$ into the $n-1$ graded component of $A \otimes A^{o p}$. But it is clear that the derivations $\delta_{Q}$ never decrease the grading, and so we cannot have $\partial_{i}=\delta_{Q}$.

On the other hand, we will now show that any "sufficiently smooth" derivation is equal to some $\delta_{Q}$. Here "sufficiently smooth" will mean that the derivation can be extended to the semi-finite algebra from [8]. As we will discuss below, we believe that this condition should be equivalent to closability.

We will use the construction from [8]; the reader is referred there for details. To avoid conflict with the notation of the current paper, we will use $\mathcal{A}_{+}, \mathcal{V}_{+}$to denote the algebras $A_{+}, V_{+}$from [8]. Note that we have an identification $A=e_{0} \mathcal{V}_{+} e_{0}$. 
Proposition 4.9. Let $\widetilde{\delta}: \mathcal{V}_{+} \rightarrow \mathcal{V}_{+} e_{0} \otimes e_{0} \mathcal{V}_{+}^{o p}$ be a derivation such that $\widetilde{\delta}_{\mathcal{A}_{+}}=0$ Let $\delta: A \rightarrow A \otimes A^{\text {op }}$ denote the restriction of $\widetilde{\delta}$; then $\delta=\delta_{Q}$ for some $Q \in \Phi$.

Proof. Recall from [8] that $\mathcal{V}_{+}$is generated by $\mathcal{A}_{+}$and $\left\{c_{n}, c_{n}^{*}: n \geq 0\right\}$, where

$$
c_{n}=\square
$$

Let

$$
\begin{aligned}
& \widetilde{\delta}\left(c_{n}\right)=\sum_{\substack{s \text { even } \\
t \text { odd }}} a_{2 n, s} \otimes b_{2 n+1, t}^{o p}, \\
& \widetilde{\delta}\left(c_{n}^{*}\right)=\sum_{\substack{s \text { odd } \\
t \text { even }}} a_{2 n+1, s} \otimes b_{2 n, t}^{o p},
\end{aligned}
$$

where $a_{m, s} \otimes b_{m, t}^{o p} \in V_{m, 0}^{(-1)^{s}}(s, 0) \otimes V_{0, m}^{+}(t, 0)$ (a slight abuse of notation, as this need not be a pure tensor).

Let $p \geq 0, q>0$ be even with $p+q=2 n$. We claim that for any $y \in P_{n}$ and any even $s$ and odd $t$ we have the following relation:

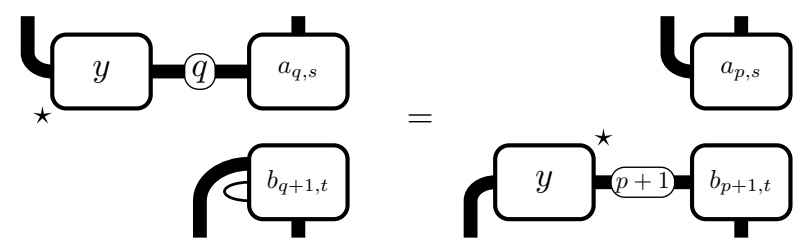

To prove this we first note that it suffices to show this for $q$ sufficiently large, which follows easily from $c_{n}=\delta \cdot e_{2 n} c_{n+1}$. We therefore assume that $q>2 s$, which will simplify the formulas which follow.

It follows from routine calculations that

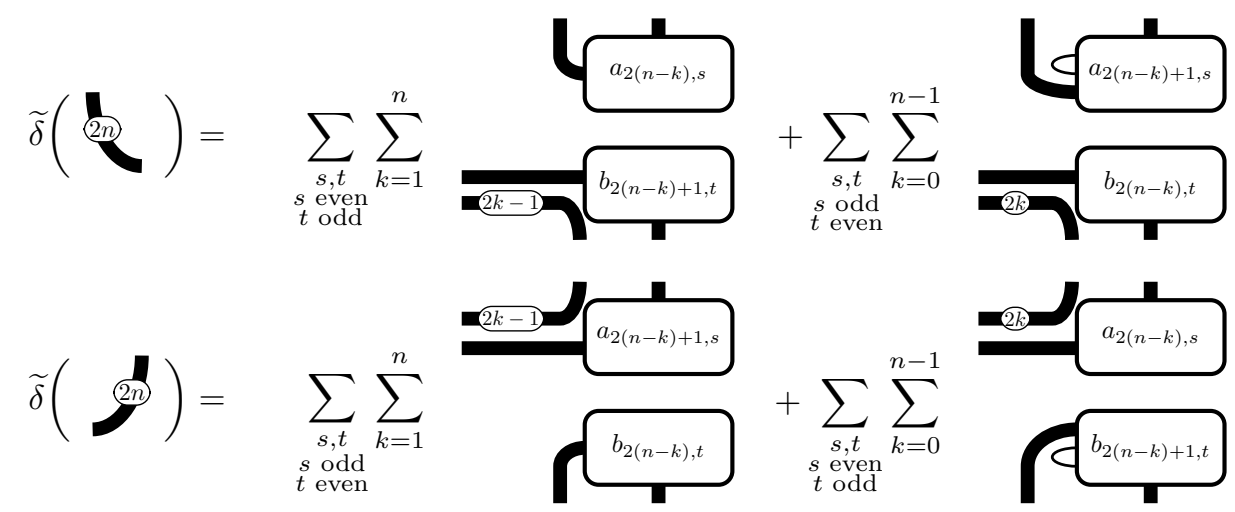

Now let $R_{s, t}$ denote the component of $\widetilde{\delta}(y)$ which lies in $V_{0,0}(p+2 s, q-1+t)$. Since we have

$$
\widetilde{\delta}(y)=\widetilde{\delta}(y),
$$


it follows from the formulas above that

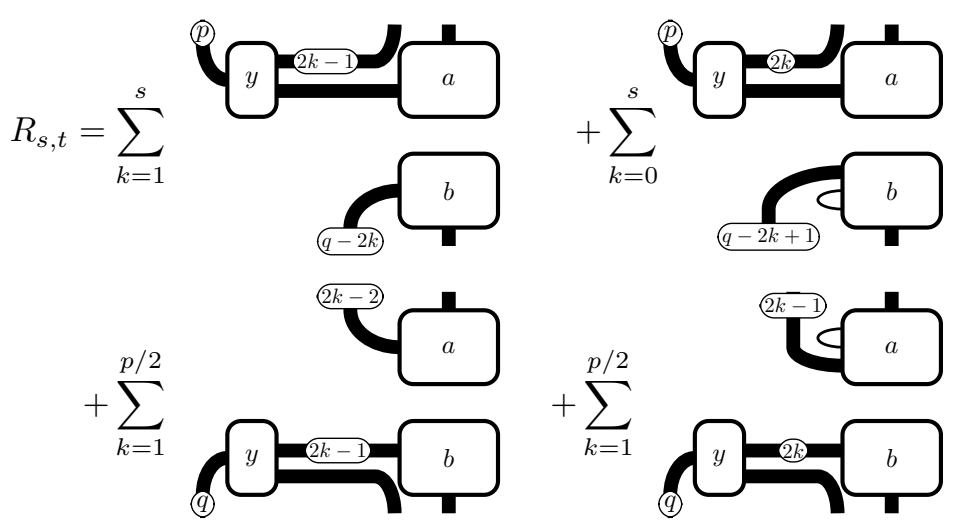

Note that we have suppressed the subscripts on $a$ and $b$, which are determined by the condition that each term has $p+2 s$ upper strings and $q-1+t$ lower strings.

On the other hand, we also have

$$
\widetilde{\delta}(y)=\widetilde{\delta}\left(\sqrt[6+1]{y} y^{(q-1}\right) .
$$

It then follows by similar computations that

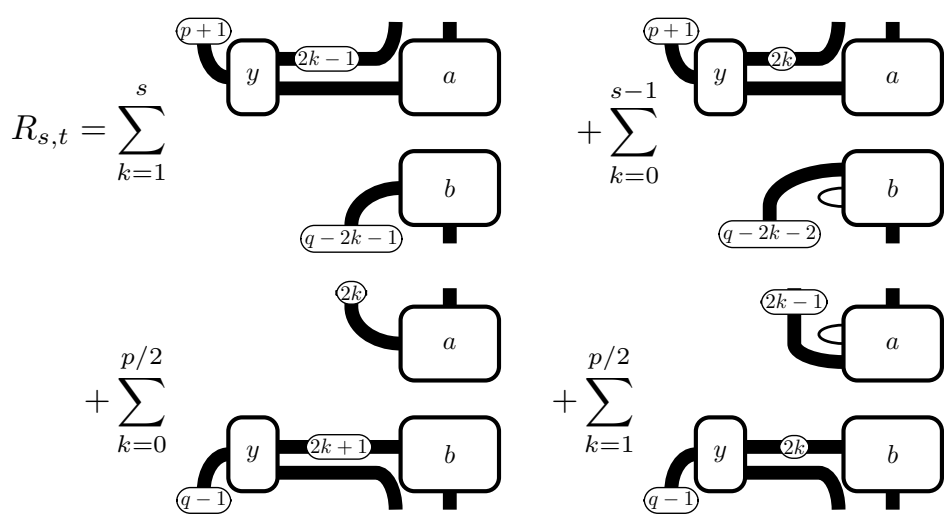

Setting these two expressions for $R_{s, t}$ equal to each other and cancelling out common terms, we obtain the desired formula.

By the same argument, if $p, q \geq 1$ are odd and $p+q=2 n$, then for any $y \in P_{n}$ and any odd $s$ and even $t$ we have:

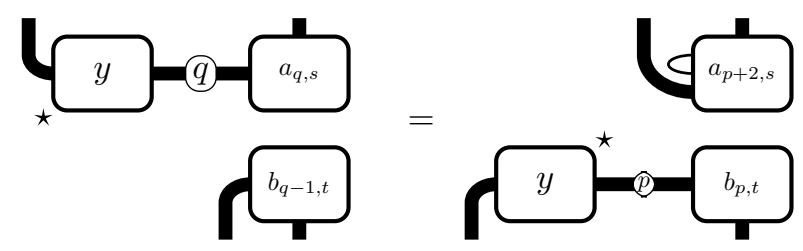


Now for $s \geq 1$ odd and $t \geq 0$ even, define $Q_{s, t} \in V_{1,0}^{-}(s, t)$ by

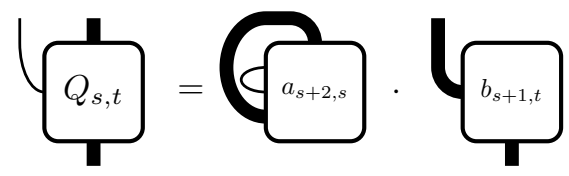

We claim that
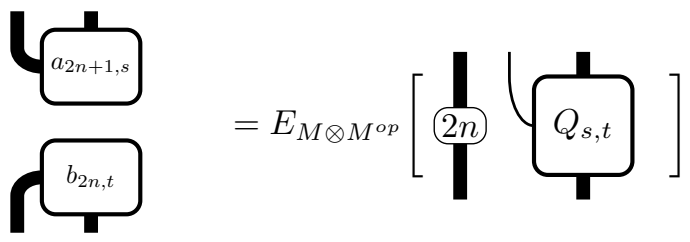

Indeed, using the relation above we see that for $x \in V_{0,2 n+1}(s, 0)$ and $y \in V_{t, 0}(2 n, 0)$ we have:

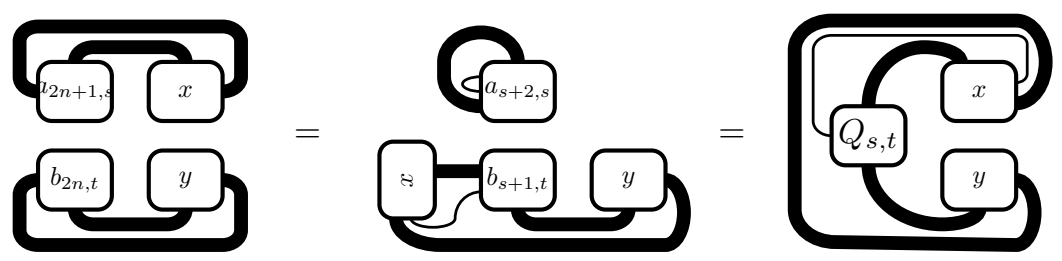

The result then follows from Lemma 4.2 .

Likewise, for $s \geq 0$ even and $t \geq 1$ odd, define

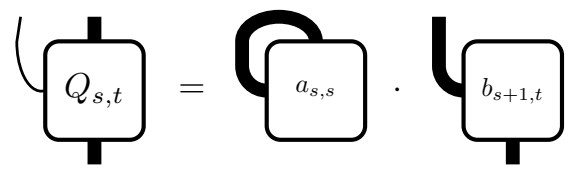

A similar argument shows that

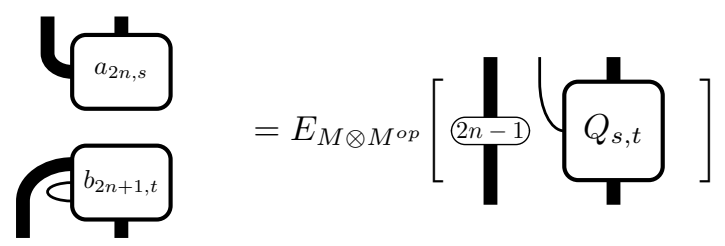

Now set $Q=\sum_{s, t} Q_{s, t}$; we claim that $\delta=\delta_{Q}$, where $\delta$ is the restriction of $\widetilde{\delta}$ to $A=e_{0} \mathcal{V}_{+} e_{0}$. Indeed, for $x \in P_{n}$ we have

$$
\delta(x)=\widetilde{\delta}(
$$

which completes the proof. 
Remark 4.10. As mentioned above, we expect that every closable derivation $\delta$ : $A \rightarrow A \otimes A^{o p}$ has an extension to a derivation $\widetilde{\delta}: \mathcal{V}_{+} \rightarrow \mathcal{V}_{+} e_{0} \otimes e_{0} \mathcal{V}_{+}^{o p}$ which vanishes on $\mathcal{A}_{+}$. To show this it would be enough to find a system of matrix units $\left\{e_{i j}\right\}$ in the completion of $\mathcal{V}_{+}$such that $e_{11}=e_{0}$, and which are smooth enough that $e_{1 i} \mathcal{V}_{+} e_{j 1}$ is contained in a holomorphic closure of $A$. Once we have such a system, we can define $\widetilde{\delta}$ by

$$
\widetilde{\delta}(a)=\sum_{i, j} e_{i 1} \bar{\delta}\left(e_{1 i} a e_{j 1}\right) e_{1 j}
$$

where $\bar{\delta}$ denotes the closure and we use crucially that $e_{1 i} a e_{j 1} \in \mathfrak{D}(\bar{\delta})$ for any $a \in \mathcal{V}_{+}$.

The existence of such a system of matrix units should in principle follow from a more careful analysis of the arguments in [8], and of the work of Dykema [4, [5]. Since we are only aiming in this section to provide some intuition for the parameter $r_{0}$, and its relation to derivations on $A$, we will avoid such technical questions. But let us point out that we do have natural extensions of the derivations $\delta_{Q}$ to $\mathcal{V}_{+}$as follows.

Proposition 4.11. If $Q \in \Phi$, then there is a derivation $\widetilde{\delta}: \mathcal{V}_{+} \rightarrow V_{+} e_{0} \otimes e_{0} \mathcal{V}_{+}^{o p}$ such that $\left.\widetilde{\delta}\right|_{\mathcal{A}_{+}}=0$ and $\left.\widetilde{\delta}\right|_{A}=\delta_{Q}$.

Proof. Let $Q=\sum_{s, t \geq 0} Q_{s, t}$, where $Q_{s, t} \in V_{1,0}^{(-1)^{s}}(s, t)$. Define $\widetilde{\delta}$ by $\left.\widetilde{\delta}\right|_{\mathcal{A}_{+}}=0$ and

$$
\begin{aligned}
& \widetilde{\delta}\left(c_{n}\right)=\sum_{\substack{s \text { even } \\
t \text { odd }}} a_{2 n, s} \otimes b_{2 n+1, t}^{\text {op }}, \\
& \widetilde{\delta}\left(c_{n}^{*}\right)=\sum_{\substack{s \text { odd } \\
t \text { even }}} a_{2 n+1, s} \otimes b_{2 n, t}^{o p},
\end{aligned}
$$

where $a_{m, s} \otimes b_{m, t}^{o p} \in V_{m, 0}^{(-1)^{s}}(s, 0) \otimes V_{0, m}^{+}(t, 0)$ are defined by

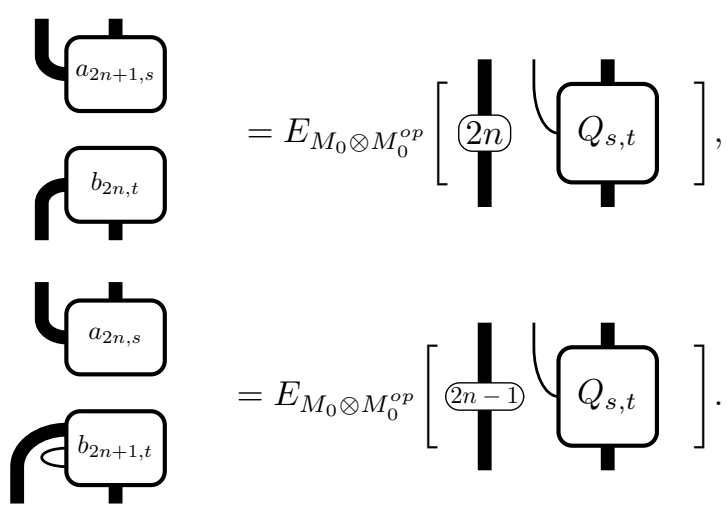

It then follows from the proof of Proposition 4.9 that $\widetilde{\delta}$ is a well-defined derivation, and that its restriction to $A$ is $\delta_{Q}$.

Remark 4.12. In Voiculescu's free analysis [21, [22, the central objects are derivations $\delta: A \rightarrow A \otimes A$ which are coassociative, i.e.

$$
(\delta \otimes \mathrm{id}) \circ \delta=(\mathrm{id} \otimes \delta) \circ \delta .
$$


Unfortunately, there do not appear to be any natural coassociative derivations among the $\delta_{Q}$ which we have considered in this section.

There is however a natural way of defining coassociative derivations on $A$ as follows. Given $Q \in P_{1}$, define $\partial_{Q}: A \rightarrow A \otimes A$ by

$$
\partial_{Q}(x)=\sum_{k=0}^{n}(\mathrm{id} \otimes \mathrm{op}) E_{M \otimes M^{o p}}\left[x_{2 k}\right]
$$

It is easy to see that $\partial_{Q}$ is coassociative, so that $\left(A, \partial_{Q}\right)$ forms a (graded) infinitesimal bialgebra [21], 1]. However these derivations do not behave well with respect to the Voiculescu trace; in particular, they do not appear to be closable.

\section{REFERENCES}

[1] Marcelo Aguiar, Infinitesimal Hopf algebras, New trends in Hopf algebra theory (La Falda, 1999), Contemp. Math., vol. 267, Amer. Math. Soc., Providence, RI, 2000, pp. 1-29, DOI 10.1090/conm/267/04262. MR1800704(2001k:16066)

[2] Alain Connes and Dimitri Shlyakhtenko, $L^{2}$-homology for von Neumann algebras, J. Reine Angew. Math. 586 (2005), 125-168, DOI 10.1515/crll.2005.2005.586.125. MR2180603 (2007b:46104)

[3] P. Di Francesco, O. Golinelli, and E. Guitter, Meanders and the Temperley-Lieb algebra, Comm. Math. Phys. 186 (1997), no. 1, 1-59, DOI 10.1007/BF02885671. MR 1462755 (99f:82028)

[4] Ken Dykema, Free products of hyperfinite von Neumann algebras and free dimension, Duke Math. J. 69 (1993), no. 1, 97-119, DOI 10.1215/S0012-7094-93-06905-0. MR.1201693 (93m:46071)

[5] Ken Dykema, Interpolated free group factors, Pacific J. Math. 163 (1994), no. 1, 123-135. MR 1256179 (95c:46103)

[6] Frederick M. Goodman, Pierre de la Harpe, and Vaughan F. R. Jones, Coxeter graphs and towers of algebras, Mathematical Sciences Research Institute Publications, vol. 14, SpringerVerlag, New York, 1989. MR999799 (91c:46082)

[7] A. Guionnet, V. F. R. Jones, and D. Shlyakhtenko, Random matrices, free probability, planar algebras and subfactors, Quanta of maths, Clay Math. Proc., vol. 11, Amer. Math. Soc., Providence, RI, 2010, pp. 201-239. MR2732052 (2012g:46094)

[8] A. Guionnet, V. Jones, and D. Shlyakhtenko, A semi-finite algebra associated to a subfactor planar algebra, J. Funct. Anal. 261 (2011), no. 5, 1345-1360, DOI 10.1016/j.jfa.2011.05.004. MR.2807103(2012j:46091)

[9] A. Guionnet, V. F. R. Jones, D. Shlyakhtenko, and P. Zinn-Justin, Loop Models, Random Matrices and Planar Algebras, Comm. Math. Phys. 316 (2012), no. 1, 45-97, DOI 10.1007/s00220-012-1573-1. MR2989453

[10] Vaughan Jones, Dimitri Shlyakhtenko, and Kevin Walker, An orthogonal approach to the subfactor of a planar algebra, Pacific J. Math. 246 (2010), no. 1, 187-197, DOI 10.2140/pjm.2010.246.187. MR2645882 (2011i:46075)

[11] Vijay Kodiyalam and V. S. Sunder, From subfactor planar algebras to subfactors, Internat. J. Math. 20 (2009), no. 10, 1207-1231, DOI 10.1142/S0129167X0900573X. MR2574313 (2011d:46129)

[12] Vijay Kodiyalam and V. S. Sunder, On the Guionnet-Jones-Shlyakhtenko construction for graphs, J. Funct. Anal. 260 (2011), no. 9, 2635-2673, DOI 10.1016/j.jfa.2011.01.018. MR:2772347 (2012b:46134)

[13] Adrian Ocneanu, Quantized groups, string algebras and Galois theory for algebras, Operator algebras and applications, Vol. 2, London Math. Soc. Lecture Note Ser., vol. 136, Cambridge Univ. Press, Cambridge, 1988, pp. 119-172. MR996454 (91k:46068)

[14] Sorin Popa, Symmetric enveloping algebras, amenability and AFD properties for subfactors, Math. Res. Lett. 1 (1994), no. 4, 409-425. MR1302385 (95i:46095) 
[15] Sorin Popa, An axiomatization of the lattice of higher relative commutants of a subfactor, Invent. Math. 120 (1995), no. 3, 427-445, DOI 10.1007/BF01241137. MR1334479|(96g:46051)

[16] Sorin Popa, Some properties of the symmetric enveloping algebra of a subfactor, with applications to amenability and property T, Doc. Math. 4 (1999), 665-744. MR.1729488 (2001c:46116)

[17] Dimitri Shlyakhtenko, Free probability, planar algebras, subfactors and random matrices, Proceedings of the International Congress of Mathematicians. Volume III, Hindustan Book Agency, New Delhi, 2010, pp. 1603-1623. MR2827857 (2012g:46096)

[18] Dimitri Shlyakhtenko, Lower estimates on microstates free entropy dimension, Anal. PDE 2 (2009), no. 2, 119-146, DOI 10.2140/apde.2009.2.119. MR2547131 (2012a:46129)

[19] Dan Voiculescu, Limit laws for random matrices and free products, Invent. Math. 104 (1991), no. 1, 201-220, DOI 10.1007/BF01245072. MR.1094052 (92d:46163)

[20] Dan Voiculescu, The analogues of entropy and of Fisher's information measure in free probability theory. V. Noncommutative Hilbert transforms, Invent. Math. 132 (1998), no. 1, 189227, DOI 10.1007/s002220050222. MR 1618636 (99d:46087)

[21] Dan Voiculescu, Free analysis questions. I. Duality transform for the coalgebra of $\partial_{X}: B$, Int. Math. Res. Not. 16 (2004), 793-822, DOI 10.1155/S1073792804132443. MR2036956 (2005a:46140)

[22] Dan-Virgil Voiculescu, Free analysis questions II: the Grassmannian completion and the series expansions at the origin, J. Reine Angew. Math. 645 (2010), 155-236, DOI 10.1515/CRELLE.2010.063. MR2673426 (2012b:46144)

Department of Mathematics, University of California, Los Angeles, Los Angeles, CALIFORNia 90095

E-mail address: curransr@math.ucla.edu

Current address: The D. E. Shaw Group, New York, New York 10036

E-mail address: Stephen.Curran@deshaw.com

Department of Mathematics, University of California, Berkeley, California 94720

E-mail address: vfr@math.berkeley.edu

Department of Mathematics, University of California, los Angeles, Los Angeles, CALifornia 90095

E-mail address: shlyakht@math.ucla.edu 